\title{
Peripheral ring opacity of the cornea
}

\author{
A. J. BRON \\ Moorfields Eye Hospital, City Road, London, E.C.I
}

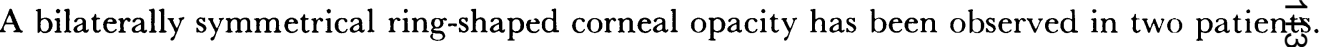
The condition is described here because of its unusual appearance and because of its appasent uniqueness in the literature.

\section{Case reports}

(I) A 59-year-old Caucasian male presented at the casualty department complaining of prickiog in the right eye. Symptoms were caused by a marginal infiltrate and this resolved on conventional therapy. A recurrence one month later also responded well.

Each cornea presented an arcus senilis and, in the zone of stroma affected by the arcus, an addition opacity could be seen. This was identical in each eye, and took the form of a striking, narrow, depge white ring in the stroma, passing forwards as a band from Descemet's to Bowman's membra\&e (Fig. I).



FIG. I Ciase

Drawing of right and

left corneae. Inse? show slit-lamp se⿳亠口冋. tions above and belores in the right eye

In slit section, except above, each band appeared as a slender wedge-shaped opacity with $\underset{9}{9}$ ts base lying on Descemet's membrane and its apex reaching forwards to Bowman's membrane. The opacity was dense at the base and faint at the apex (Fig. 2, opposite). Between the I I to I o'clळ k positions, the rings were very faint in each eye and sloped inwards and forwards at an angle in eक्क्र $h$


no opacity peripheral to the white ring at the level of Descemet's membrane apart from that associated with the arcus senilis. 

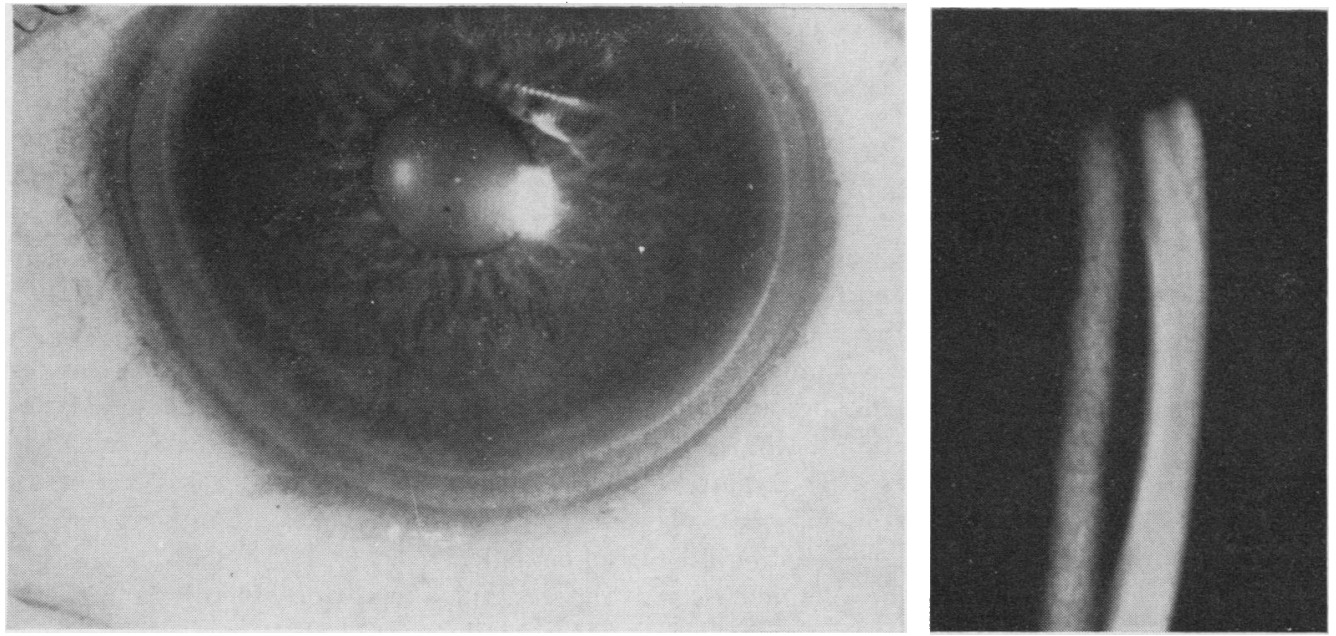

FIG. 2a Case I. Slit-lamp photograph of right cornea, showing ring opacity. $\quad \times 2$

FIG. 2b Case I. Slit-lamp photograph of right cornea. Slit section showing density of opacity on Descemet's membrane. $\times 4$

On gonioscopy both angles were wide with normal Schwalbe's lines and in places the site of the white rings could be visualized central in position to Schwalbe's lines and not projecting into the anterior chamber.

The visual acuity was $6 / 5$ in either eye, the central cornea was clear and corneal sensation was normal. The remainder of the anterior segments were normal, as were the visual fields, ocular tension, and posterior segments. There was no history of allergy and no history of drugs or chemicals. The patient's family originated in Russia, and no family members were available for study. Full blood picture, serum protein and electrophoresis, and blood cholesterol were normal. The Wassermann reaction was negative.

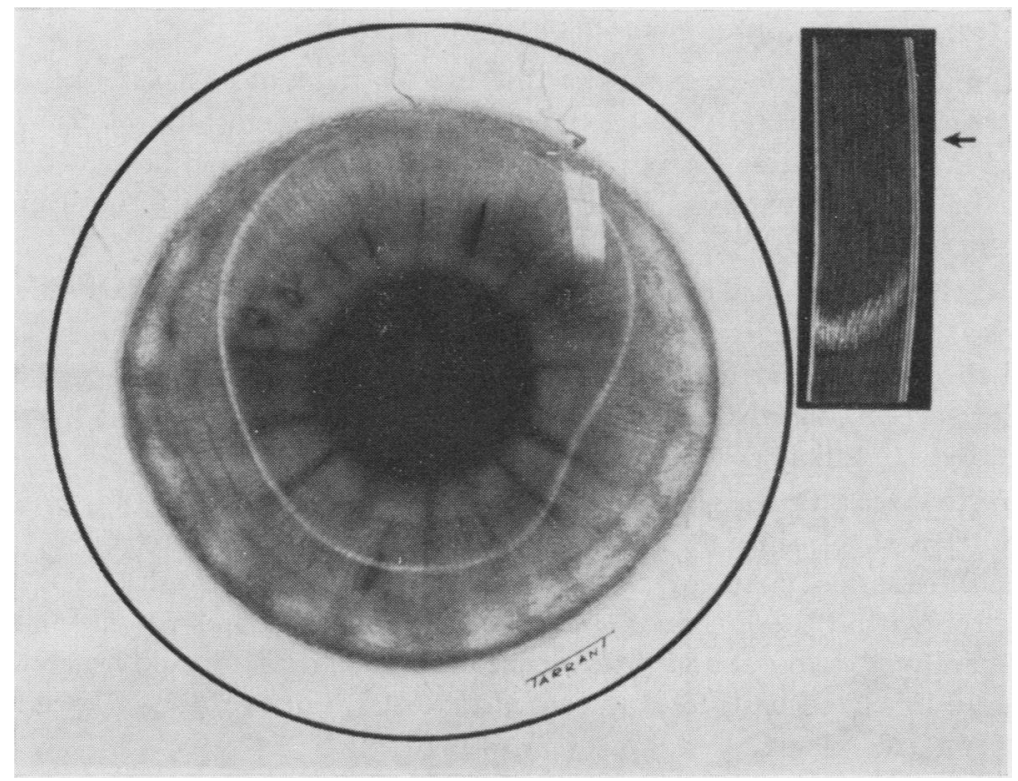

FIG. 3 Case 2. Drawing of right cornea, showing ring opacity of cornea 
(2) A 66-year-old Caucasian female presented at the casualty deparment complaining of pain a产 discharge in the right eye. She was found to have a marginal corneal infiltrate. This healed rapi on routine therapy.

As an incidental finding, each cornea exhibited oval ring-shaped opacities which were symmetrical in the two eyes (Fig. 3). Each ring comprised a narrow stromal band lying on Bowman's membragte. The ring was white in colour and of fibrillar texture, its density greatest next to Descemet's membra In slit section it could be seen to trace a gentle curve from behind forwards, so that its anterior es lay central to its posterior edge. In each eye the ring was approximately $2 \mathrm{~mm}$. from the limbus, am̃ nearer the limbus above and below. Each ring was very faint above.

Both corneae showed anterior and posterior crocodile shagreen outside the ring and broad horizontal Hudson-Staehli lines within it. These lines showed marked degenerative changes about their centres. Also, central to the rings were multiple linear greyish streaks, mainly on Descemछ̈"s membrane but also scattered sparsely in the stroma. These lay vertically, horizontally, or obliquef.

Corneal sensation was normal. Uncorrected visual acuity was 6/9 in both eyes. The rest of $\mathbb{W}_{\mathrm{e}}$ eye appeared normal, including the filtration angle.

The patient had no allergic history. She was taking trinitrini tablets for angina, and had suffered a coronary thrombosis to years before. Her family were of Huguenot origin. Her daughter had had treatment for eye disease, but the nature of this was unknown.

\section{Discussion}

The similarities between the peripheral ring opacities seen in these two patients have be $\overrightarrow{e n}$ brought out in the clinical descriptions. The differences are trivial, in that the rings seencin the second case are more central and less dense than in the first. The appearances ffe distinctive, but might be confused with two other common bilateral corneal conditio posterior and anterior embryotoxon.

In posterior embryotoxon the ring-shaped opacity represents the thickened and axialifidisplaced anterior border ring of Schwalbe. This may be seen by slit-lamp examination alone, but gonioscopy shows how the material projects into the anterior chamber. I I made up of the hypertrophied collagen of the anterior border ring (Hogan and Zimme man, I 962). This feature and the absence of a stromal opacity in posterior embryotoxin distinguish it from the condition described here.

In anterior embryotoxon, or arcus senilis, the opacity has an internal and exterial margin anteriorly, but posteriorly it extends out as far as Schwalbe's line. The arcuşis broader and the margins less sharply defined than in the condition described here. M్ㅐㄹ important, the stromal opacity of arcus senilis is dense in relation to both limiting meonbranes, less dense in the stroma between (Duke-Elder, r965).

There are other ring-shaped disorders of the cornea such as Coats's white ring (Miller a플 Gordon, I950), annular heredodystrophy of the corneal endothelium (François and Eves, 1960), and the ring-shaped deposits associated with hereditary spherocytosis (Dalgleisb, I965). They are manifestly different from the ring opacity described here and do nigt enter the differential diagnosis.

It remains to discuss the origins of the peripheral ring opacities of the cornea. The lateral symmetry of the rings and absence of vessels suggest a dystrophic or degenerat basis for their formation. An early onset is usually given as a requisite for the diagnosis of corneal dystrophy, but there is no a priori reason why a dystrophy should not have a late onset. Certainly a late onset and the absence of a visual defect would lessen the chancegof establishing an inherited basis for any corneal disorder, but this does not exclude the possibility. 
The patients were aged 59 and 66 years, and a further patient with the same condition and of similar age, has also been observed in the same hospital (not seen by the author and therefore not described). Thus all three patients are in the age group of degenerative corneal disorders.

One last possibility is that the rings are an immune type of stromal ring representing the location of stromal damage at the site of previous antigen-antibody interaction. Both patients had marginal keratitis which is thought by some to have an immune basis. Such immune rings, concentric with the limbus, may be produced in animals by the experimental introduction of antigen into the centre of the cornea in the presence of circulating antibody against this antigen. The acute ring is made up of a zone of infiltrating leucocytes in the area of the antigen-antibody precipitation (Germuth, Maumenee, Pratt-Johnson, Senterfit, Van Arnam, and Pollack, I958). It has been suggested that such rings may occur in cases of keratitis (Breebaart, I963). They centre around the primary corneal lesion and are therefore not necessarily concentric with the limbus. These rings disappear when the primary focus disappears. The stromal rings seen after herpes simplex and herpes zoster keratitis may be of this nature. Although the possibility of an immune basis for the peripheral corneal rings is intriguing, the absence of any central stromal disturbance in Case I, at least, is against this.

Thus it would seem best to regard the condition of peripheral ring opacity of the cornea as a degeneration until other evidence accumulates.

\section{Summary}

The appearance of a new form of corneal degeneration is described. The salient features are of a well-defined narrow band opacity of the peripheral stroma in the form of a ring. The ring is indistinct above. It is dense white on Descemet's membrane but fainter as it passes forward to Bowman's membrane. There is no vascularisation of the cornea and vision is normal.

My thanks are due to Mr. C. A. G. Cook and Prof. B. R. Jones for permission to report these two cases, and also to Mr. T. A. Tarrant for the corneal drawings.

\section{References}

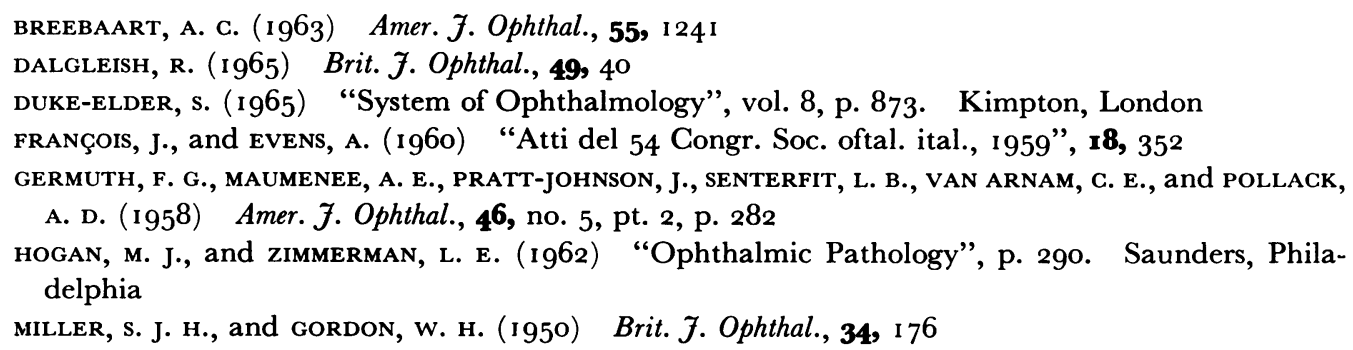

